Literatur

- Argyris, C. / Schön, D.: Organizational learning: A Theory of Action Perspective; Reading, Mass., 1978

- Bateson, G.: Ökologie des Geistes; Frankfurt a.M., 1992

- Bartölke, K.: Organisationsentwicklung für entwicklungsfähige Organisationsmitglieder; In: Kappler, E. (Hrsg.):Unternehmensstruktur und Unternehmensentwicklung; Freiburg i. B., 1980, S. 319-344

- Berger, U. / Bernhard-Mehlich, I.: Die Verhaltenswissenschaftliche Entscheidungstheorie; In: Kieser, A., 1993, S. 127-159

- Champbell, D.: Variation and selective retention in socio-cultural evolution; In: General Systems, 1969, S. $69 \mathrm{ff}$

- Geißler, H.: Vom Lernen in der Organisation zum Lernen der Organisation; In: Sattelberger, T., 1994, S. 79-96

- Hannan, M. T. / Freeman, J.: The population ecology of organizations; In: American Journal of Sociology, 1977, S. $929 \mathrm{ff}$

- Hejl, P. M.: Konstruktion der sozialen Konstruktion; In: Einführung in den Konstruktivismus; München, 1985, S. 109-146

- Kieser, A. (Hrsg.): Organisationstheorien; Stuttgart, 1993

- Kirsch, W.: Kommunikatives Handels, Autopoiese, Rationalität; München, 1992

- Luhmann, N.: Soziale Systeme; Frankfurt a.M., 1993

- Lutz, Chr.; Kommunikation - Kern der Selbstorganisation: Unternehmensführung im Informationszeitalter; In: Sattelberger, T., 1994, S. $97-110$

- Maturana, H. / Varela, F. J.: Der Baum der Erkenntnis; Bern/München, 1987

- Türk, K.: Neuere Entwicklungen in der Organisationsforschung; Stuttgart, 1989

- Pautzke, G.: Die Evolution der organisatorischen Wissensbasis; München, 1989

- Probst, G. J. B.: Selbstorganisation - Ordnungsprozesse in sozialen Systemen aus ganzheitlicher Sicht; Berlin/Hamburg, 1987

- Probst, G. J. B./Scheuss, R.-W.: Resultat von Organisieren und Selbstorganisation; In: ZfO Heft $8 / 1984$, S. $480-488$

- Rieckmann, H.: Organisationsentwicklung - von der Euphorie zu den Grenzen; In: Sattelberger, T., 1994, S. 125-144

- Sattelberger, T.: Die lernende Organisation im Spannungsfeld von Strategie, Struktur und Kultur; In: Sattelberger, T., 1994, S. 11-56

- Sattelberger, T. (Hrsg.): Die lernende Organisation; Wiesbaden, 1994

- Ulrich, H. / Probst, G. J. B.: Anleitung zum ganzheitlichen Denken und Handeln; Bern/Stuttgart, 1988

- Varela, J. F. / Thompson, E. / Rosch, E.; Der Mittlere Weg der Erkenntnis; München, 1992

- Willke, H.: Strategien der Intervention in autonome Systeme; In: Baecker, D. (Hrsg.): Theorie als Passion; Frankfurt a. M., 1987, S. 333-361

- Wollnik, M.: Das Verhältnis von Organisationsstruktur und Organisationskultur; In: Dülfer, E. (Hrsg.): Organisationskultur; Stuttgart, 1988, S. $49-76$

\title{
Unternehmen als Lernende Systeme
}

\section{Konkurrenz \\ der Schlagworte}

Aufgrund der Wettbewerbssituation, die sich auf vielen Märkten dramatisch zugespitzt hat (Stichworte: Saturierung, Internationalisierung, Rezession), befinden sich Unternehmen auf dem Weg zu tiefgreifenden technisch-organisatorischen Umstrukturierungen und Umorientierungen. Es gilt, Wege zu finden, schneller und treffender als die Konkurrenz Impulse aus der Umwelt aufzunehmen, zu interpretieren und in möglichst sach- und kundengerechte Lösungen umzusetzen.

Immer schon hat es eine Konkurrenz der »LeitKonzepte « in der Management»lehre« (1) gegeben. Das kompetitive Element war aber dadurch relativiert, daß die Ansätze in regelrechten Modewellen zeitlich aufeinanderfolgen und es eine verschärfte Konkurrenz wohl nur in den Übergangsphasen gegeben hat. Die Konzepte Werksgemeinschaft, Human Relations, Betriebliche Partnerschaft, Kooperative Führung, Soziale Verantwortung des Unternehmens, Humanisierung der Arbeit, Organisationsentwicklung oder Unternehmenskultur spiegeln im wesentlichen das Spektrum der in Rede stehenden Leit-Kategorien von den 20er bis zu den späten 80er Jahren dieses Jahrhunderts wider (2).

Nie hat es aber zeitgleich einen derartigen Wettbewerb um die kategoriale Führerschaft auf dem lukrativen Markt der Managementkonzepte gegeben wie heute. Zwar gibt es mit der »Lean Production « inklusive aller Abwandlungen einen Favoriten oder Spitzenreiter; aber sie hält keineswegs ein Monopol. Das Feld der Konkurrenten ist dicht: Human Resource Management, Total Quality Management, Kaizen (Kontinuierlicher VerbesserungsprozeB), Reengieering, Virtuelle Organisation sowie: Lernende Organisation. Inhaltlich unterscheiden sich die Ansätze nur wenig voneinander. Zum konstanten Kern gehören etwa die folgenden Merkmale:

- anti-tayloristisch, de-zentralistisch, team(arbeits)-orientiert;

- Funktionalisierung der Kompetenzen und der Kreativität der Mitarbeiter/innen zu kontinuierlichen Verbesserungsprozessen;

- Rückverlagerung der Qualitätsverantwortung an den Ort der Leistungserstellung;

- Orientierung an kundenbezogenen Geschäftsprozessen statt Abteilungsdenken; segmentierte, modulare Fertigungsorganisation; internes Kunden-LieferantenVerhältnis.

Die Leit-Konzepte entstammen aber unterschiedlichen »Schulen « und setzen wenigstens zum Teil verschiedene Schwerpunkte. Im folgenden soll der Ansatz einer näheren Betrachtung unterzogen werden.

\section{Theoretischer Bezugsrahmen Lernender Organisationen}

Die Konzepte zur Lernenden Organisation (3) leiten sich aus der Vorstellung ab, daß nicht nur Individuen lernen, also sich Verhaltensänderungen aufgrund von Erfahrungen einstellen, sondern auch Unternehmen diese Möglichkeit besitzen. Lernen in Organisationen beinhaltet alle interaktiven Prozesse, die die Fähigkeit zur Variation, zur bewußten und erfolgreichen Veränderung der unternehmenseigenen operativen Programme besitzen und somit ein Potential der Anpassung und Weiterentwicklung von Organisationen schaffen.

Organisationales Lernen bedeutet nicht nur die Summe des Wissens der Organisationsmitglieder. Individuelles Wissen wird in der Organisation gespeichert, sukzessiv abstrahiert und in das organisationale System implementiert. Als Speichermedien fungieren Leitlinien, Arbeitsanweisungen, Mythen und Kultur. Organisationales Wissen besteht unabhängig von bestimmten Mitgliedern und verläßt somit auch bei einem Personalwechsel das Unternehmen nicht. Jede Organisation besitzt diese Art von Wissen und bestimmt damit institutionelles Wissen.

Organisationales Wissen verändert sich aber nur durch den Zugang zu individuellem Wissen. Der Austausch der Ergebnisse individueller Lernprozesse muß daher innerhalb des Unternehmens gewährleistet sein. Kommunikation stellt dabei das entscheidende Bindeglied zwischen individuellen und organisationalen Wissensbasen dar. Die Organisation muß so gestaltet sein, daß ein interner Erfahrungsaustausch möglich wird bzw. stattfindet. »Der Lernprozeß des einzelnen muß vermittelbar, auswertbar und im sozialen System mit Lernprozessen anderer Organisationsmitglieder verknüpfbar sein« (4)

Gruppen oder Teamstrukturen ùnterstützen durch ihre Transformations- und ihre kollektive Lernfunktion diesen Vorgang. Als Transformationsfunktion bezeichnet man die Möglichkeit, individuelles Lernen in das organisationale System einzubringen, um mit Hilfe der kollektiven Lernfunktion von Gruppenstrukturen eine andere Lernebene zu erreichen.

Insgesamt muß die Organisationsstruktur eines lernenden Unternehmens aber nicht nur so gestaltet sein, daß Kommunikationsprozesse ablaufen können, sondern auch eine anschließende Reflexion über die erhaltenen Informationen stattfindet. Lernorganisationen besitzen deshalb »Freiräume « für hierarchiefreie Dialoge, um einen effektiven Reflexionsprozeß zu gewährleisten. Lernprozesse gestalten sich demnach besser ohne Hierarchie in einer Arena des Vertrauens und der Gleichberechtigung.

Der Prozeß organisationalen Lernens findet in unterschiedlicher Art der Veränderung des 
Unternehmens seinen Abschluß. Die Modifikation kann sich dabei auf die Struktur und Arbeitsabläufe einer Organisation beziehen oder aber auf den Gesamtrahmen der Organisation. Die Transformation des Bezugsrahmens der Organisation ist kein einmaliger Lernproze $ß$, sondern ein kontinuierlicher. Ziel ist es, daß der Proze $ß$ des Wandels in und außerhalb der Organisation, Widersprüche und Unsicherheit in der Organisationsrealität von dem Unternehmen und seinen Mitgliedern verinnerlicht und angenommen werden.

Die Umsetzung des Konzepts der Lernorganisation stellt sich als außerordentlich langwierig und noch in den »Kinderschuhen steckend « dar. Es versteht sich von selbst, daß lernförderliche Elemente wie Hierarchieabbau und Gleichberechtigung in erwerbswirtschaftlichen Organisationen auf enorme Widerstände stoßen. Dennoch werden in der Literatur einige Versuche, Unternehmen zu Lernenden Organisationen zu entwickeln, beschrieben. Dabei handelt es sich vorwiegend um die Veränderung bestehender Organisationsstrukturen, die Erweiterung von Schlüsselqualifikationen und von Lernpotentialen, sowie den Aufbau von Vertrauensorganisationen, in denen Gleichberechtigung und Kommunikation gelten.

\section{Anzeige}

\section{Wir bieten Beteiligungen an:}

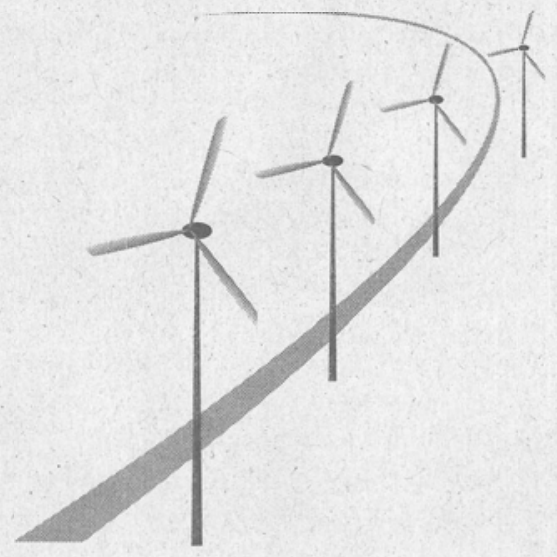

- Windkraftprojekt Sottrum

- Fonds

Spieka-Neufeld/Sottrum/Beckum

Für weitere Informationen rufen Sie uns einfach an oder schreiben uns unter dem Stichwort »IÖW"

\section{ENERGIEKONTOR Windkraft GmbH}

Planung und Betrieb von Windkraftanlagen Stresemannstr. 46 - 27570 Bremerhaven . Tel. 0471/140-72 Fax 0471/140-99
Praxisbeispiele für den Versuch,

ABB führte 1992 ein Kundenorientierungsprogramm ein. 1994 hat ein Viertel der Belegschaft an diesem Weiterbildungsprogramm teilgenommen. Damit sind 9000 Mitarbeiter einer Orauf eine bestimmte Unternehmenstrategie geschult worden. Ehrgeiziges Ziel des ternehmens ist die Schulung der Gesamtskräfte von $\mathrm{ABB}$ werden zusätzlich im quartalsmäßige Anfertigung von Reports rungen. Die Auswertung, Aufbereitung und Weitergabe dieser Informationen und der einzelnen Prozeßbeobachtungen werden durch exrne Beraterteams übernommen.

Hilfe für die Probleme im täglichen rbeitsproze $\beta$ gibt eine eigens gegründete Firsich vor allem mit den Bereichen Pro. Unternehmens begleitend fördern.

FESTO unterstreicht die Forderung, daß alle Mitarbeiter die Idee des Lernunternehmens kennen müssen. Fähigkeiten der Kommunikation, Teamarbeit, Problemlösungsund Realisierungsmöglichkeiten und Konfliktbewältigung werden gefördert, um individuelles und organisationales Lernen aufeinander abzustimmen. Seit 1987 ist das Lernunternehmen bei FESTO Thema in den Führungsetagen. Die einzelnen Führungskräfte im Gesamtunternehmen wurden sukzessiv mit dem Gedanken, FESTO als Lernende Organisation zu sehen, vertraut gemacht. Unterstützt wurden diese Maßnahmen durch mehrsprachig veröffentlichte Broschüren wie »Lernen ist Zukunft «

Seit 1989 wird die Implementation durch Task Forces im Unternehmen begleitet. Ab 1990 führte die FESTO Academy Informationsveranstaltungen über die Lernorganisation durch. Die Mitarbeiter hatten jetzt die Möglichkeit, den in diesen Veranstaltungen vorhandenen Diskussionsspielraum mit ihren eigenen Ideen und Vorstellungen auszufüllen. Seit 1992 existiert ein Gesprächskreis »Lernunternehmen« auf der oberen Managementebene, der durch die FESTO Academy beratend unterstïtzt wird.

Durch Veränderungen in der Arbeitsstrukturierung in Form von Team- und Gruppenkonzepten wurden Lernzellen im Unternehmen eingerichtet. Zur Zeit entstehen im gesamten Unternehmen konkrete Projekte zur Lernorganisation. Führungskräfte berichten in zeitlich festgelegten Abständen über ihre Erfahrungen und Lernprozesse, um anderen Mitarbeitern die Chance der Wissenserweiterung zu geben. Jeder Mitarbeiter soll sich aktiv am Gedanken des Lernunternehmens beteiligen können. Daher gibt es zahlreiche Seminare, Gruppentreffen und Foren, die eine offene Kommunikation und die Simulation von Problemlösungen ermöglichen.

Diese eher auf die tägliche Praxis des Unternehmens bezogene Bildungsarbeit wird zusätzlich durch Veranstaltungen zu den theoretischen Grundlagen der Lernenden Organisation begleitet. Prozeßbegleitung, Organisationsentwicklung, Konfliktmanagement oder aber auch Philosophieschulungen stehen in diesem Bereich auf der Tagesordnung. FESTO versucht durch dieses sehr umfangreiche Lern-Lehr-Angebot, daß sich die Organisationsmitglieder in den Lernprozeß einbringen, um ein kreatives und flexibles Unternehmen der Zukunft entwickeln zu lassen.

Gleichwohl sollen diese Beispiele nicht über die Tatsache hinwegtäuschen, daß das Konzept der Lernenden Organisation generell noch ein Wunschtraum ist und vielfach auch bleiben wird. Letztendlich hat man sich mit verheißungsvollen Proklamationen wesentlich leichter getan, als mit realen Veränderungen.

Peter Wengelowski, Thomas Breisig, Oldenburg

Anmerkungen

(1) Die Anführungszeichen in Management»lehre « deshalb, weil es mehr die populärwissenschaftliche Literatur sowie die Partizipanten am heißumkämpften Unternehmensberatungs-Markt sind, die solche LeitKonzepte »puschen«. Damit ist eine - stets zu beobachtende - Rezeption durch die Betriebswirtschaftslehre als der »zuständigen« Wissenschaft nicht ausgeschlossen.

(2) Vgl. Breisig, Thomas: Betriebliche Sozialtechniken. Handbuch für Betriebsrat und Personalwesen, Frankfurt a. M. u. Neuwied 1990, S. 14 f.

(3) In der Literatur gibt es kein einheitliches Konzept der Lernenden Organisation. Die Begriffe organisationales Lernen und Lernorganisation werden dabei als synonym verwandt. (Siehe dazu Veröffentlichungen von: Pautzke, Reinhardt, Geißler, Sattelberger usw..)

(4) Pawlowsky, Peter: Betriebliche Qualifikationsstrategien und organisationales Lernen, S.220, in: Conrad, Peter; Steahle, Wolfgang: Managementforschung 2, Berlin u. a. 1992, S. 177-237. 
(c) 20I0 Authors; licensee IÖW and oekom verlag. This is an article distributed under the terms of the Creative Commons Attribution Non-Commercial No Derivates License (http://creativecommons.org/licenses/by-nc-nd/3.o/), which permits unrestricted use, distribution, and reproduction in any medium, provided the original work is properly cited. 